\title{
Critical points and transitions in an electric power transmission model for cascading failure blackouts
}

\author{
B. A. Carreras and V. E. Lynch \\ Oak Ridge National Laboratory, Oak Ridge, Tennessee 37831 \\ I. Dobson \\ ECE Department, University of Wisconsin, Madison, Wisconsin 53706 \\ D. E. Newman \\ Physics Department, University of Alaska, Fairbanks, Alaska 99775
}

(Received 1 March 2002; accepted 10 July 2002; published 9 September 2002)

Cascading failures in large-scale electric power transmission systems are an important cause of blackouts. Analysis of North American blackout data has revealed power law (algebraic) tails in the blackout size probability distribution which suggests a dynamical origin. With this observation as motivation, we examine cascading failure in a simplified transmission system model as load power demand is increased. The model represents generators, loads, the transmission line network, and the operating limits on these components. Two types of critical points are identified and are characterized by transmission line flow limits and generator capability limits, respectively. Results are obtained for tree networks of a regular form and a more realistic 118-node network. It is found that operation near critical points can produce power law tails in the blackout size probability distribution similar to those observed. The complex nature of the solution space due to the interaction of the two critical points is examined. (c) 2002 American Institute of Physics. [DOI: $10.1063 / 1.1505810]$

From the analysis of a 15-year time series of North American electric power transmission system blackouts, we have found that the frequency distribution of the blackout sizes does not decrease exponentially with the size of the blackout, but rather has a power law tail. The existence of a power tail suggests that the North American power system has been operated near a critical point. To see if this is possible, here we explore the critical points of a simple blackout model that incorporates circuit equations and a process through which outages of lines may happen. In spite of the simplifications, this is a complex problem. Understanding the different transition points and the characteristic properties of the distribution function of the blackouts near these points offers a first step in devising a dynamical model for the power transmission systems.

\section{INTRODUCTION}

In spite of technological progress and great investments to ensure a secure supply of electric energy, blackouts of the U.S. electric transmission grid are not uncommon. In the last three decades, blackouts have been happening on average of one every 13 days. ${ }^{1,2}$ Furthermore, analyses of 15 years of North American blackout data show a probability distribution of blackout sizes has a power tail. ${ }^{3-5}$ The power tails indicate that large blackouts are much more likely than might be expected from Gaussian statistics. Understanding and analyzing these power tails is important because of the enormous cost to society of large blackouts.

Detailed analysis of large blackouts has shown that they involve cascading events in which a triggering failure produces a sequence of secondary failures that lead to blackout of a large area of the grid. ${ }^{6}$ Cascading events and power tails in the probability distribution function are suggestive of a complex system operating close to a critical point. It is therefore important to explore this possibility for electric power systems.

General approaches from the perspective of network structure have been developed in studying properties of power system networks. ${ }^{7,8}$ We have proposed ${ }^{9-11}$ an electric power transmission model to study the dynamics of blackouts. This model (The model of Refs. 9-11 includes slow dynamics of load increase and network upgrade as well as fast dynamics of individual cascading blackouts. In this paper, we assume a fixed network and only study the fast dynamics of the model of Refs. 9-11) captures features of cascading outages and is consistent with the standard dc power flow equations ${ }^{12}$ for a given network structure. In this paper, we analyze the critical points of this model as a function of the increasing power demand. Transition points have been identified for other types of networks, like traffic models, ${ }^{13}$ computer networks, ${ }^{14}$ and neural networks. ${ }^{15}$

In the present calculations, we have considered two types of networks. One type is the idealized tree network such as the one shown in Fig. 1. These networks are useful because their symmetry allows the use of very few free parameters and the properties of the network can be studied by increasing its size in a self-similar manner. Although the tree network is an artificial network with more regularity than a real power network, the three lines incident on each node is approximately the average for large power networks. We also 


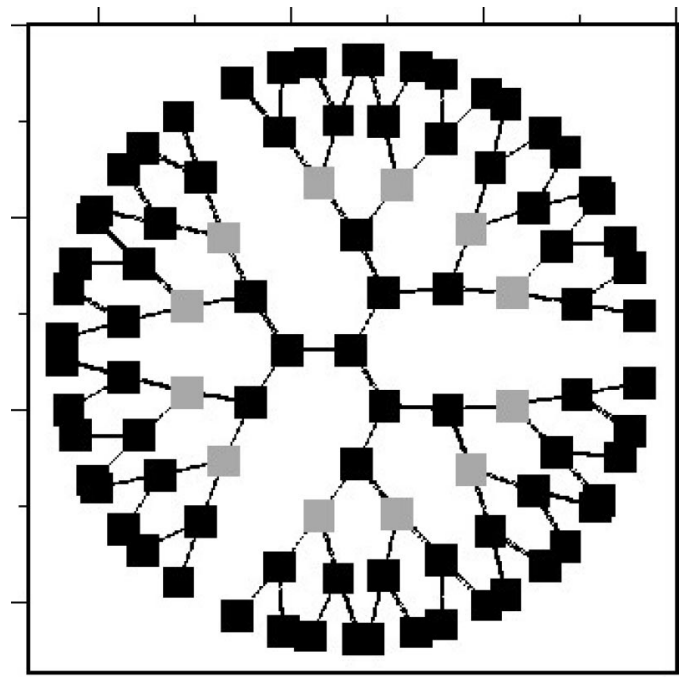

FIG. 1. A 94-node tree network with 12 generators (gray squares) and 82 loads (black squares).

have considered the IEEE 118-bus network ${ }^{13}$ shown in Fig. 2 to test whether the main results obtained for the ideal networks are relevant for more realistic networks.

The paper is organized as follows. Section II describes the model for the electric power transmission that we use to study blackouts. The solutions of this model applied to ideal tree networks are discussed in Sec. III. As the power demand increases, several transition points are identified. The structure of the solutions and transition regions are presented in Sec. IV. Section V discusses the effect of fluctuations in the power demand and calculates the probability distribution function of the blackout size. Application of this model to a more realistic network is presented in Sec. VI. Finally, the conclusions are given in Sec. VII.

\section{ELECTRIC POWER TRANSMISSION MODEL}

We use a simple model to describe the electric power transmission network as a set of nodes interconnected by transmission lines. The network nodes represent loads (L), generators $(\mathrm{G})$, or combinations of load and generation. The

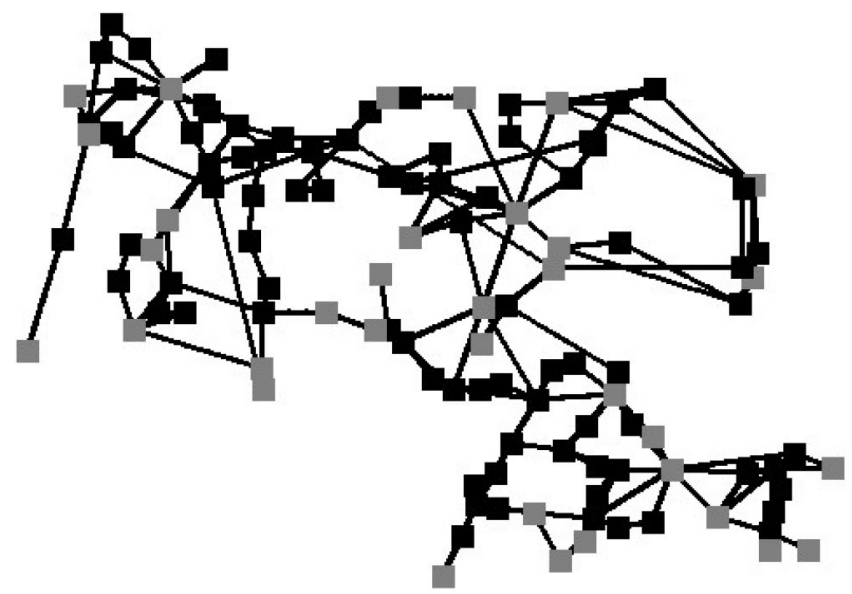

FIG. 2. Diagram of the IEEE 118 bus network. Generators are gray squares and loads are the black squares. network nodes are characterized by the input power, $P_{i}$, which is positive for generators and negative for loads, and, in case of generators, the maximum power that a generator can supply, $P_{i}^{\max }$. Each network transmission line connects two nodes, $i$ and $j$, and is characterized by the power flow through the line, $F_{i j}$, the maximum power flow that it can carry, $F_{i j}^{\max }$, and the impedance of the line, $z_{i j}$. This model allows the consideration of any interconnected network with $N_{N}=N_{G}+N_{L}$ nodes and $N_{1}$ lines, where $N_{G}$ is the number of generators and $N_{L}$ is the number of loads.

The "dc power flow" equations are used to study the power flow through the network; they give a linear relationship between the power flowing through the lines and the power input at the nodes. This approach is a standard way of analyzing a power transmission system ${ }^{12}$ and it is equivalent to a linearized version of the more common problem of solving for the voltages and currents in a circuit. The dc power flow equations can be written as

$$
F=A P,
$$

where $F$ is a vector whose $N_{1}$ components are the power flows through the lines, $F_{i j}, P$ is a vector whose $N_{N}-1$ components are the input power of each node, $P_{i}$, except the reference generator, $P_{0}$, and $A$ is a constant matrix, whose elements can be calculated in terms of the impedance of the lines. More detail on Eq. (1) is given in the Appendix and in Ref. 12.

For a given load power demand and the grid parameters defined above, the system of equations (1) does not have a unique solution. There are many ways of choosing a combination of generator powers to satisfy a given load demand. Therefore, to find the solution to this system, we chose an optimum combination of generator powers. Optimization of a real power transmission system accounts for many factors, from safe operation to economic gain. Here we use a standard optimization approach, ${ }^{16-18}$ and we solve the power flow equations, Eq. (1) while minimizing the simple cost function:

$$
\text { Cost }=\sum_{i \in G} P_{i}(t)-W \sum_{j \in L} P_{j}(t)
$$

In this model, we assume that all generators run at the same cost and all loads have the same priority to be served. However, we set up a high price for load shed by setting $W=100$. The minimization of the cost function is done with the following constraints.

(1) Limits on the generator power: $0 \leqslant P_{i} \leqslant P_{i}^{\max } i \in G$;

(2) The loads must remain such and cannot generate power: $P_{j} \leqslant 0 j \in L$;

(3) Power flow through the lines is limited: $\left|F_{i j}\right| \leqslant F_{i j}^{\max }$;

(4) The total power generated and consumed must balance: $\Sigma_{i \in G \cup L} P_{i}=0$.

This optimization problem is a standard linear programming (LP) problem. ${ }^{16,17}$ It is numerically solved using the simplex method as implemented in Ref. 19.

It is useful to introduce the quantity 


$$
M_{i j} \equiv \frac{F_{i j}}{F_{i j}^{\max }} .
$$

This quantity is the fraction of overloading of the line connecting the nodes $i$ and $j$. We use the quantities $M_{i j}$ and the power produced by each generator to describe the solution of the optimization problem. A line with $M_{i j}<1$ still has margin to carry more power. We consider that a line is overloaded if the power flow through this line is within $1 \%$ of $F_{i j}^{\max }$.

The entire process has several sources of nonlinearity. The constrained optimization used to solve Eq. (1) introduces nonlinearity because the active constraint can change. Looking at a sequence of solutions as a function of the total power load, we see that they are piecewise linear. This is the type of nonlinearity introduced by constraints. The lines that overload are detected by the threshold condition $M_{i j}=1$, and the line outage is implemented by changing the structure of equations and constraints; and all of these processes are nonlinear.

A cascading overload may start if one or more lines are overloaded in the solution of the linear programming problem. In this situation, we assume that there is a probability, $p_{1}$, that an overloaded line will suffer an outage. When a solution is found, the overloaded lines of the solution are tested for possible outages. If there is one or more line outages, we multiply the line impedance by a large number, $\kappa_{1}$, and divide its corresponding $F_{i j}^{\max }$ by another large number, $\kappa_{2}$. In this way, there is practically no power flow through this line. This method models well the effect of a line outage and avoids the singularity in the matrix that would result from removing the outage line. Once the power flow through the lines is reduced, a new solution is then calculated. This process can lead to multiple iterations, and the process continues until a solution is found with no more line outages. This cascading effect introduces another nonlinearity into the problem. The overall effect of the process is to generate a possible cascade of line outages that is consistent with the network constraints and optimization.

\section{SOLUTION OF THE POWER FLOW EQUATIONS FOR IDEAL NETWORKS}

Most of the results presented in this paper are based on tree networks. Although the tree networks are artificial networks with more regularity than a real power network, the three lines incident on each node is approximately the average for large power networks. In a real power network, the generation is dispersed in a variable manner throughout the network. In order to study a more ordered case, the generators in the tree networks are placed at nodes in the third generation level. An example of a tree network with 94 nodes is shown in Fig. 1. For all the tree networks considered, we keep the generators at the nodes indicated in Fig. 1. As we increase the size of the network, that is the number of nodes, we add links to two more loads for all the loads at the edge of the network. If we call each family of added loads a generation, the example in Fig. 1 has five generations of nodes. The number of nodes of a tree network as a function of the number of generations is $N_{N}=3 \times 2^{n}-2$. Because of

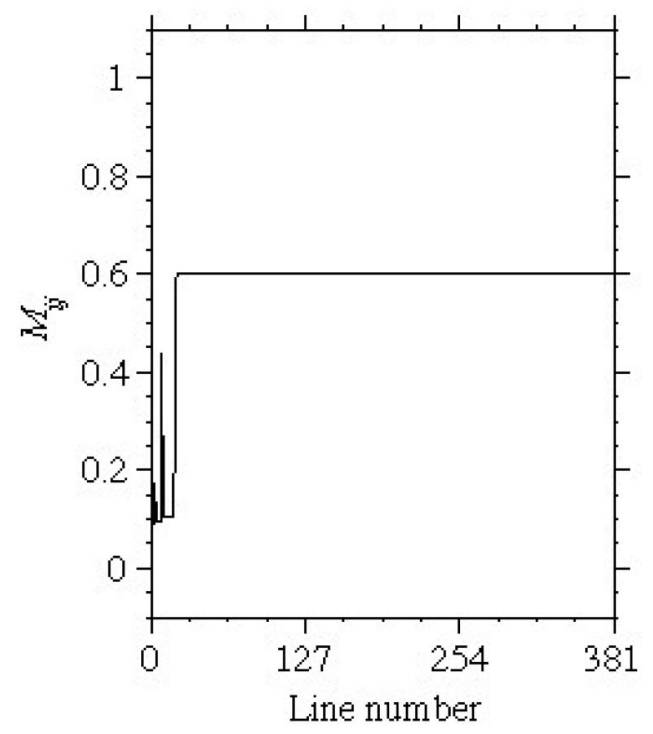

FIG. 3. Fraction of overload of the lines in the 382 node tree network operating at $P_{D} / P_{C}=0.3$, well below any of the limits of the system.

the network structure and the equal impedance of the lines, it is logical to have the maximum power flow of a line in generation $k$ to decrease as $2^{(3-k)}$. That is, the maximum power flow of lines in generation $k+1$ is half of the maximum power flow of lines in generation $k$. Note that the generators are located in the third generation. For the calculations presented here, we consider tree networks with 46, 94, 190, and 382 nodes.

Because of the symmetry and simplicity of these networks, we can work with a single control parameter, the total load power demand. We generate a sequence of solutions of the power flow equations for increasing values of the power demand. To be able to reduce the parameters to a single parameter, we take the loads to be equal to the averaged total power demand per load times a random number $r$, such that $2-\gamma \leqslant r \leqslant \gamma$, where $0 \leqslant \gamma \leqslant 2$. This random fluctuation of the loads allow us to explore different solutions for a given power demand and carry out statistical analysis of these solutions. First, we look for solution at very low power demand and without load fluctuations so that $\gamma=1$. Here, low power means low compared with the total generation capacity of the system, $P_{C}=\Sigma_{j \in G} P_{j}^{\max }$, and low enough to avoid any line overload. Under these conditions the solution is relatively simple. For all lines in the rings outside the ring of generators, the value of $M_{i j}$ is the same. This is just a consequence of our choice of values for $F_{i j}^{\max }$ and the symmetry of the system. For a particular example with $N_{N}=382$ and $P_{D} / P_{C}=0.3$, the values of $M_{i j}$ for all lines are plotted in Fig. 3. The lines outside the generator ring have line numbers greater than 12 and have $M_{i j}=0.601$. The lines in the inner region of the network have smaller values because of the redundancy of lines in this region. In spite of the symmetry of the system, an optimal solution does not have the same power output for all generators. Figure 4 shows the distribution of the power output among the generators. We can see that the power output of generator 12 is practically zero and that generator 11 has somewhat reduced power; all the other 


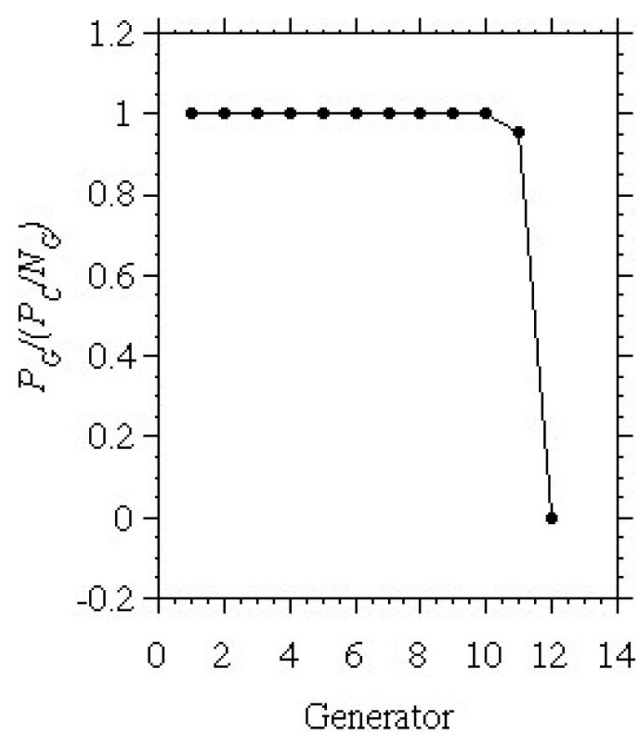

FIG. 4. Fraction of the power produced by the 12 generators in the 382 node tree network operating at $P_{D} / P_{C}=0.3$, well below any of the limits of the system.

generators operate at maximum capacity. In this type of solution, some generators are being kept as backup generators. Therefore, the LP optimization has the effect of operating the system in an inhomogeneous way, in spite of its symmetry. Because the power demand for all loads is the same, so is the power delivered to each load. A list of detailed parameters used in the calculation for those networks are given in Table I.

In this low power demand regime, the system is analytically soluble. For a network with $n$ generations of nodes and without load power fluctuations, the power demand per load is $P_{D} /\left(3 \times 2^{n}-14\right)$. Therefore, the power flow through the lines that connect nodes of the generation $k-1$ to nodes of the generation $k$ is

$$
F_{i j}=\frac{2\left(2^{n-3}-1\right)}{2^{k-3}\left(3 \times 2^{n}-14\right)} P_{D} .
$$

Having the power flows in all lines, the problem is essentially solved.

As the power demand increases, but stays small in the sense previously described, the solution is qualitatively the same. In this case, the values of $M_{i j}$ increase linearly with the power demand, as can be seen from Eq. (4).

\section{TRANSITION POINTS FOR AN IDEAL NETWORK}

As the power demand continues to increase, the model has several transition points. These transitions represent a change in the character of the solutions. These transitions can be characterized by two complementary measures of the blackout size. One of these measures is the load shed. In situations in which the power demand cannot be met by the generators, either because of insufficient capacity or because of a transmission line outage, the only way of finding a solution is by shedding load and partially or totally blacking out some nodes. In this case, the power demand is not met and the power served is lower than the demand. In what follows, we use the load shed divided by the power demand, $P_{S} / P_{D}$, as a measure of blackout size. The other measure of blackout size is the number of line outages in the final solution.

The different transitions are caused by different limits in the power system. The limits can be grouped in two types:

(1) Limits set by the available power generation. It is clear that the system cannot supply more power than $P_{C}$, the total maximum installed generator capacity.

(2) Limits set by the transmission capacity of the grid. Each line has a maximum power flow that it can carry, $F_{i j}^{\max }$. This maximum sets the limit for each line and, as a result, limits the total capacity of the network.

An example with two of these limits is shown in Fig. 5. For a tree network with 382 nodes (12 generators and 370 loads), we increase load power demand by increasing all loads at the same rate. In this example, the load demand increase is continuous, and we have not included random fluctuation in the load demands $(\gamma=1)$. As the power demand reaches the total generator capacity, $P_{D} / P_{C}=1$, and load shedding begins. As the demand continues to increase, all power above $P_{C}$ is shed. The nodes in the outermost ring of the network are progressively blacked out. When $P_{D} / P_{C}=1.45$, the power flow in some lines reaches the line power flow limit, and some line outages are produced. These line outages disconnect parts of the network from the generators and more nodes are blacked out. These blackouts further increase the load power shed.

Why is there a second transition even after the total power served is kept constant and is therefore independent of the level of demand? The reason is that the individual loads increase, and the power shed is not uniform over all loads. Therefore, even if the total power served is constant, the power delivered to some of the loads is increased as the total

TABLE I. Values of the parameters used for the tree networks. All lines have impedance $z=1$.

\begin{tabular}{|c|c|c|c|c|c|c|c|c|c|}
\hline$N_{N}$ & $P_{L}$ & $P_{G}$ & $\begin{array}{l}F^{\max } \\
n=0\end{array}$ & $\begin{array}{l}F^{\max } \\
n=1\end{array}$ & $\begin{array}{l}F^{\max } \\
n=2\end{array}$ & $\begin{array}{l}F^{\max } \\
n=3\end{array}$ & $\begin{array}{l}F^{\max } \\
n=4\end{array}$ & $\begin{array}{l}F^{\max } \\
n=5\end{array}$ & $\begin{array}{l}F^{\max } \\
n=6\end{array}$ \\
\hline 46.000 & -74 & 2623.9 & 15620 & 7748.7 & 3812.9 & 1844.9 & & & \\
\hline 94.000 & -74 & 2623.9 & 15620 & 7748.7 & 3812.9 & 1844.9 & 860.97 & & \\
\hline 190.00 & -74 & 2623.9 & 15620 & 7748.7 & 3812.9 & 1844.9 & 860.97 & 368.99 & \\
\hline 384.00 & -74 & 2623.9 & 15620 & 7748.7 & 3812.9 & 1844.9 & 860.97 & 368.99 & 123.00 \\
\hline
\end{tabular}




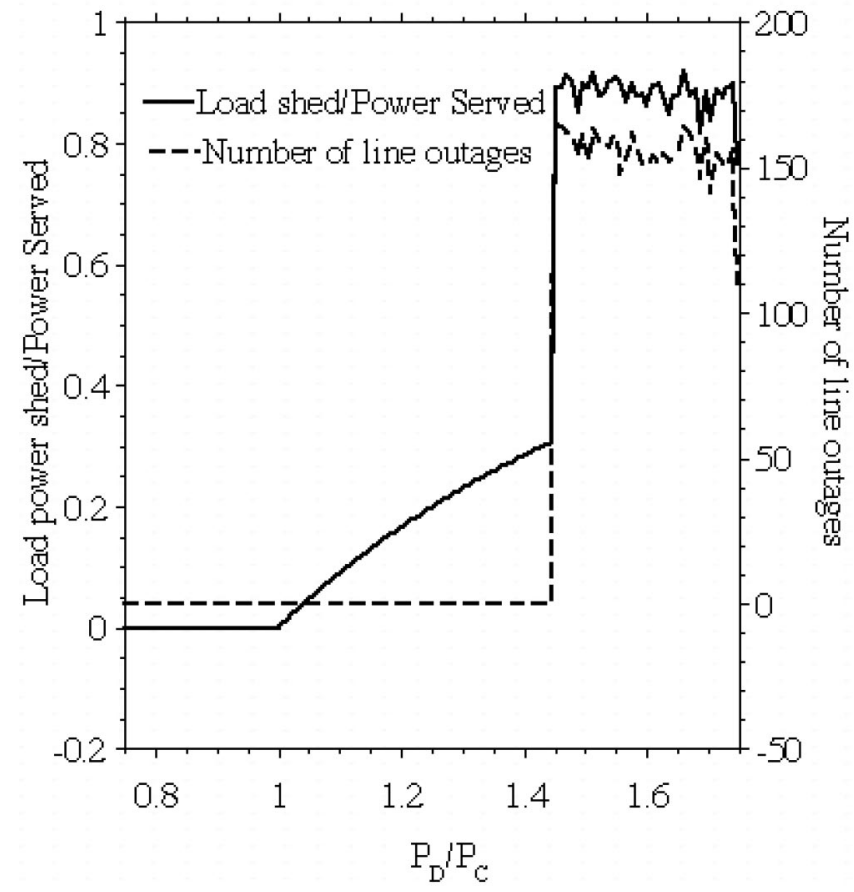

FIG. 5. Normalized power shed and number of line outages for a tree network with 382 nodes as a function of power demand.

demand increases while others are blacked out. That increase in power demand to those loads leads to overloaded lines connected to them and possible line outages. The second transition point occurs at the same value of the power demand. This transition occurs even in the absence of the first critical point because it depends on the power of individual loads and the maximum power flow that the lines connecting them can carry. These results come from studying a sequence of cases under the same conditions but without random load fluctuations. The important point is that the first transition point is a function of the total power demand, while the second transition point depends on the local value of the loads near the lines that are closer to overload. Furthermore, we have chosen the network parameters in such a way that most lines reach their limit for the same value of the power demand. In a general, inhomogeneous network, that is not the case, and the second transition breaks into multiple transitions.

For the calculation shown in Fig. 5, we have used the power demand as the control parameter, and we have done a scan starting with all load nodes having the same power loads and no fluctuations. We can look in more detail at the solutions in the region between the two transition thresholds. In this region, the solutions are well behaved and have a continuous character as a function of the power demand. Because there is power shedding, the $M_{i j}$ of some lines is now lower than the others because some loads are at nearly zero power. This example is shown in Fig. 6 for a case with $P_{D} / P_{C}=1.04$. Because the system is working at full capacity, all generators deliver their maximum power. As the power demand continues to increase, the system reaches the second threshold at $P_{D} / P_{C}=1.45$. At this value of the power demand, several lines reach their maximum loading of $M_{i j}$

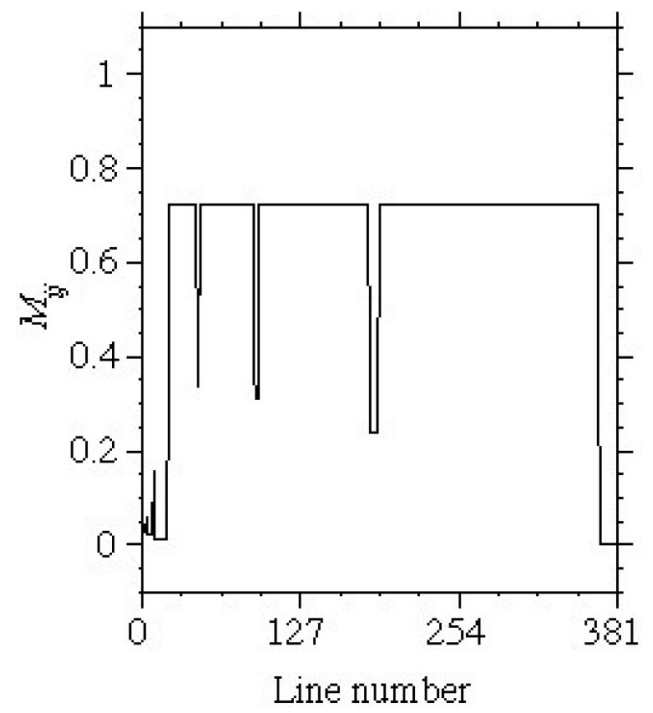

FIG. 6. Fraction of overload of the lines in the 382 node tree network operating at $P_{D} / P_{C}=1.04$, just above the maximum generator power limit but below the limits of any of the lines.

$=1$, as shown in Fig 7. To identify this transition point, it is useful to introduce $M_{\max } \equiv \max M_{i j}$. Then, the second transi$i j$ tion in Fig. 5 is given by $M_{\max }=1$. Above this threshold, there are multiple outages, the power shed is large, and the value of $M_{i j}$ in the few operating lines is low. An example of this erratic distribution of values of $M_{i j}$ is shown in Fig. 8 for $P_{D} / P_{C}=1.73$.

When the second threshold at $P_{D} / P_{C}=1.45$ is crossed, the solution does not appear to be continuous in the power demand. We have done the calculation for $p_{1}=1$. That is, all overloaded lines suffer outages. In this case the problem is in principle deterministic, but the solutions behave erratically above this threshold. This behavior can be better seen by plotting $M_{i j}$ in a two-dimensional plot as a function of power demand and line number. This plot is shown in Fig. 9 (for the

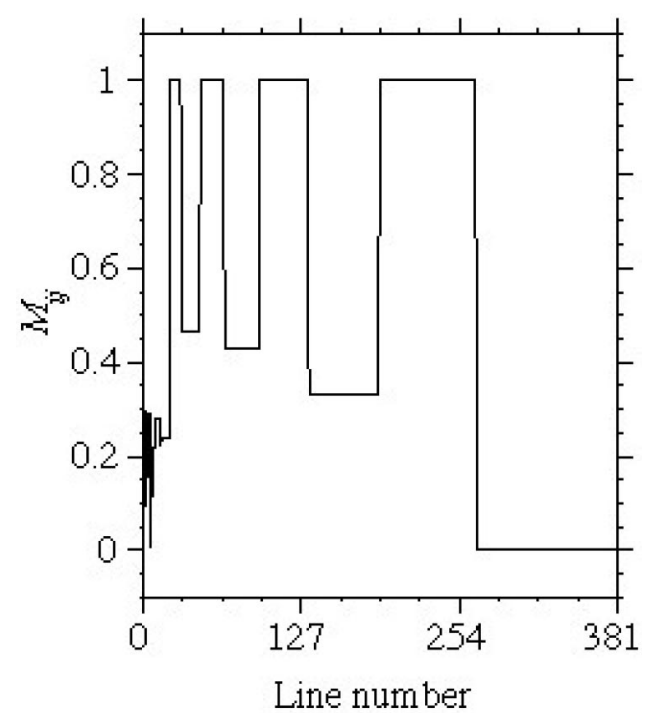

FIG. 7. Fraction of overload of the lines in the 382 node tree network operating at $P_{D} / P_{C}=1.45$, just at the limit of a set of transmission lines. 


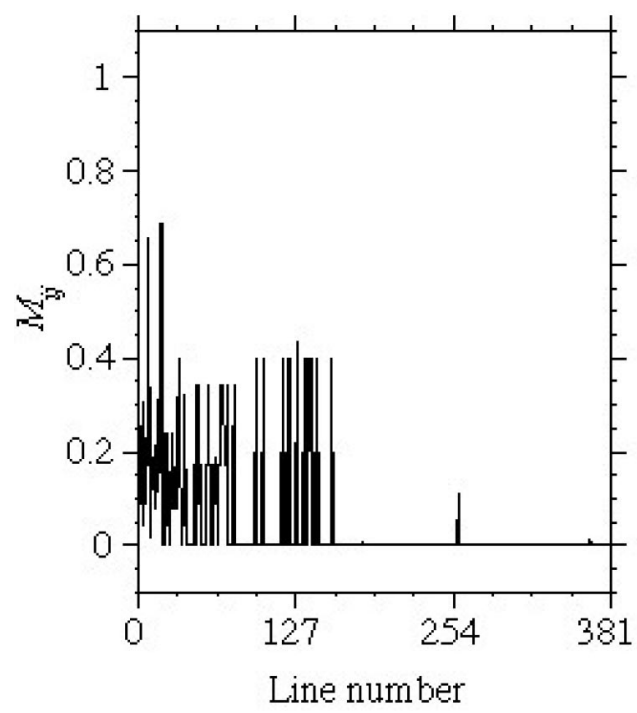

FIG. 8. Fraction of overload of the lines in the 382 node tree network operating at $P_{D} / P_{C}=1.73$, above the limit of the transmission lines.

same case as Fig. 5). There is a uniform pattern of solutions below the second threshold, and a complex pattern of solutions above the second threshold. In the region above the second threshold the solutions are not really discontinuous with the power demand. If we look at Fig. 9 with increased resolution, we see a sequence of bands of solutions. Within each band the solutions are continuous with the power demand. However, every time that a new line hits its limit, the solution changes. Small variations in the power demand cause new lines to reach their limits and that causes the apparent erratic behavior of the solutions.
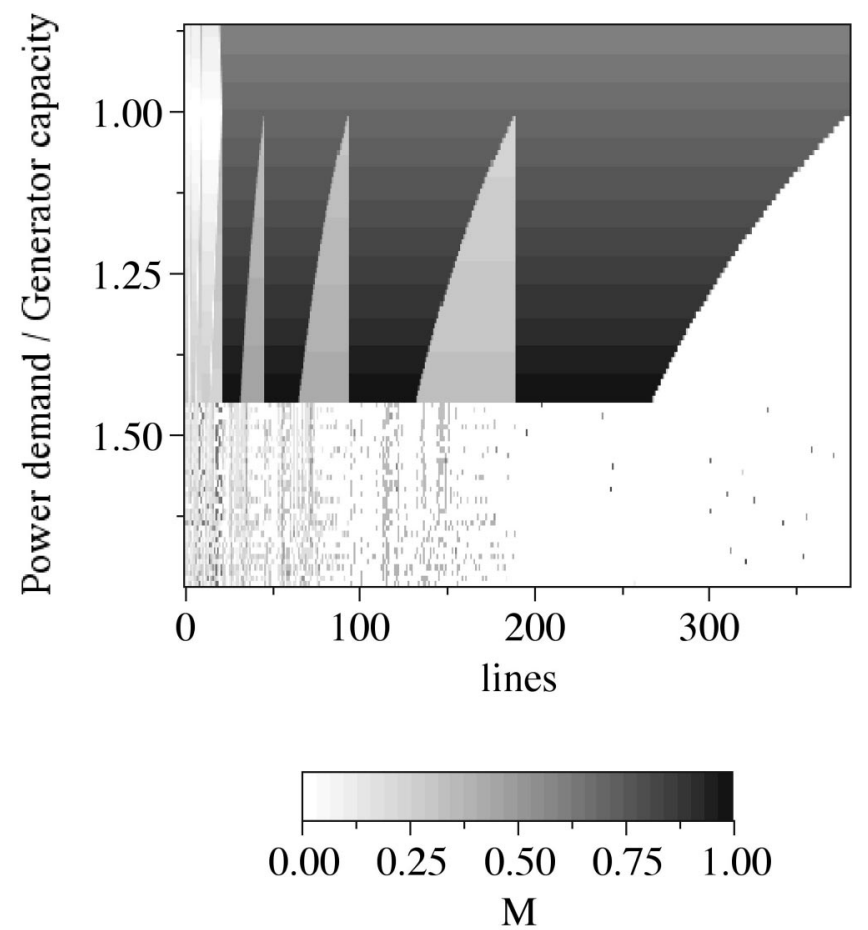

FIG. 9. Two-dimensional plot of the fraction of overload of the lines in the 382 node tree network as a function of line and power demand.

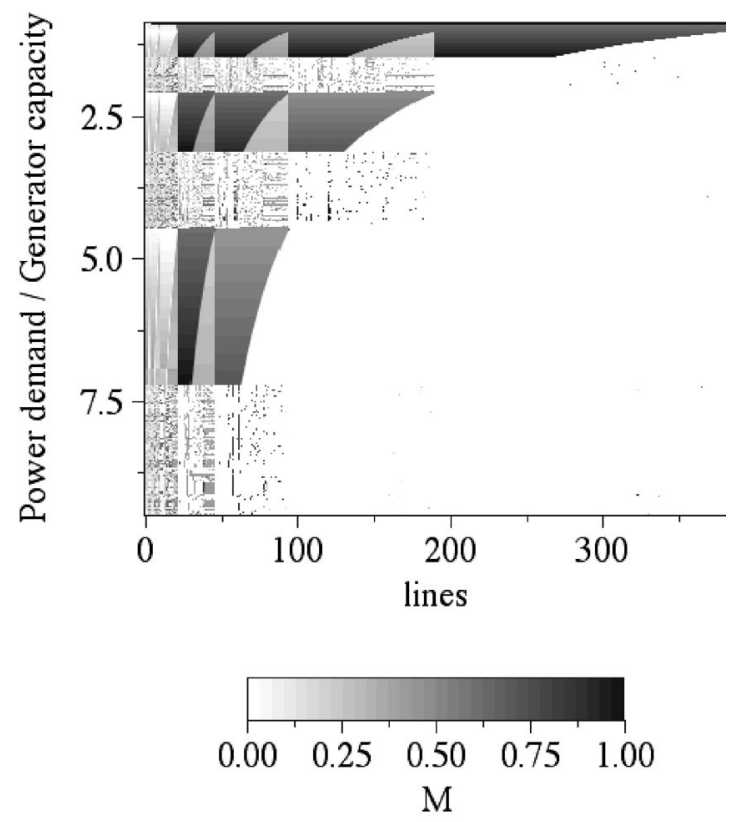

FIG. 10. The same plot as Fig. 9, but for an extended range of power demands.

The symmetry of the network results in a peculiar behavior of the thresholds. As the power demand continues to increase above the values shown in Fig. 5, it reaches a point at which all the loads on the outermost ring of the system are blacked out. At this point the system behaves as a tree network with 192 nodes, and an ordered solution is found. Now all lines have power flow below their maximum possible value, and the solutions behave like the ones in the region $1 \leqslant P_{D} / P_{C} \leqslant 1.45$. This situation continues until another set of lines becomes overloaded and the system transitions to erratic solutions. In Fig. 10, we show a plot like the one in Fig. 9, but extending to larger values of power demand. We can see alternating bands of erratic and organized solutions. Therefore, there are many possible transition points depending on the two conditions listed above and the symmetry of the network.

The properties of the transitions for $M_{\max }=1$ depend on the value of $p_{1}$, the probability that an overloaded line will suffer an outage. If $p_{1}=0$, there are no line outages, the $M_{\max }=1$ transition point no longer exists, and the load shed is a continuous function of the power demand. However, for $p_{1}=1$, all overloaded lines suffer outages. As we have already indicated, this is the value of $p_{1}$ used in the calculation shown in Fig. 9 and the transition is characterized by a discontinuous jump in the load shed. In Fig. 11, we show examples of transitions for different values of $p_{1}$. For values of $p_{1}$ in the interval $(0,1)$, for instance for $p_{1}=0.1$, we have an intermediate situation with the jump in the load shed at the transition point being smaller than in the case with $p_{1}=1$.

In these calculations, we have chosen the parameters so that the power generation limit is reached for lower power demands than the line limits. The reason for this choice is to get a clear separation between the different transitions. However, in general, the transitions are not organized in any particular way, and the way that they occur depends on a mul- 


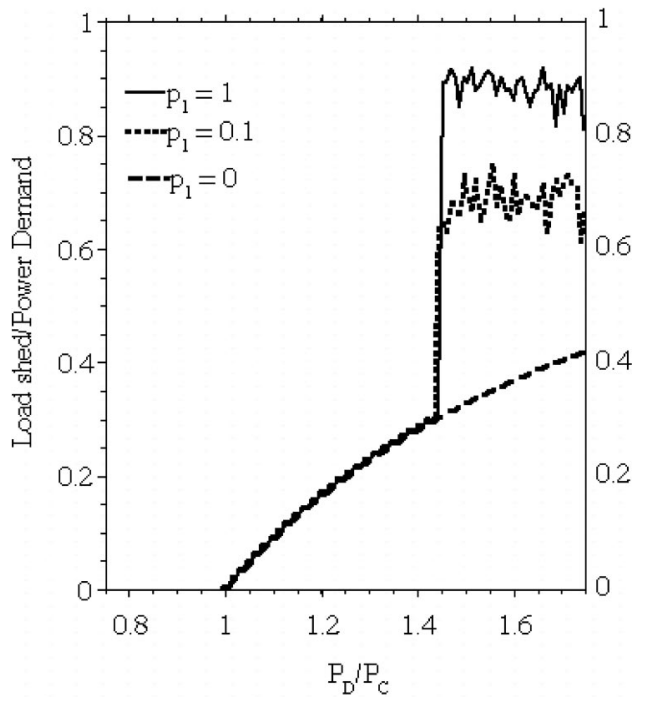

FIG. 11. Normalized power shed for a tree network with 382 nodes as a function of load power demand. Results for three different values of the probability for overloaded lines to outage are plotted.

tiplicity of parameters. The separation between the transition points may disappear when the load values fluctuate as discussed in the next section.

There are several possible numerical implementations of the optimization algorithm to solve the power transmission model. The solutions in the ordered bands are found to be independent of the solver used and so are the transition points. However, the solutions in the erratic bands may depend on the numerical method. This possibility is not surprising because the solution depends on the order that the constraints are applied, and there is irreversibility in the cascading process.

\section{EFFECT OF LOAD FLUCTUATIONS NEAR THE TRANSITION POINTS}

To understand the statistical properties of the solution of the power transmission problem near the transitions, it is interesting to introduce fluctuations in the values of the load around an averaged value given by the power demand per load. The load fluctuations are controlled by the parameter $\gamma$ as described in Sec. III. We no longer necessarily apply the random fluctuations to each node independently of the others. We group nodes by regions and vary equally all the nodes in a given region. The reason for this grouping is to simulate weather effects that normally are not limited to a single power distribution center, but rather affect to a whole region of the country. For a given value of $\gamma$, the standard deviation of the fluctuation induced in the total power demand is $\sigma=(\gamma-1) /\left(2 \sqrt{N_{F}}\right) P_{D}$. Here $N_{F}$ is the number of independent regions in the network. When we operate at a value of $P_{D} / P_{C}$ close to the generator limit, the load fluctuations may reach the generator limit. Furthermore, if $\sigma$ is large enough, the fluctuations may hit both the generator limit and some of the transition points associated with $M_{\max }=1$. In this situation, it is interesting to analyze the properties of the solutions when $P_{D} / P_{C}<1$, and we vary the parameter $\gamma$.

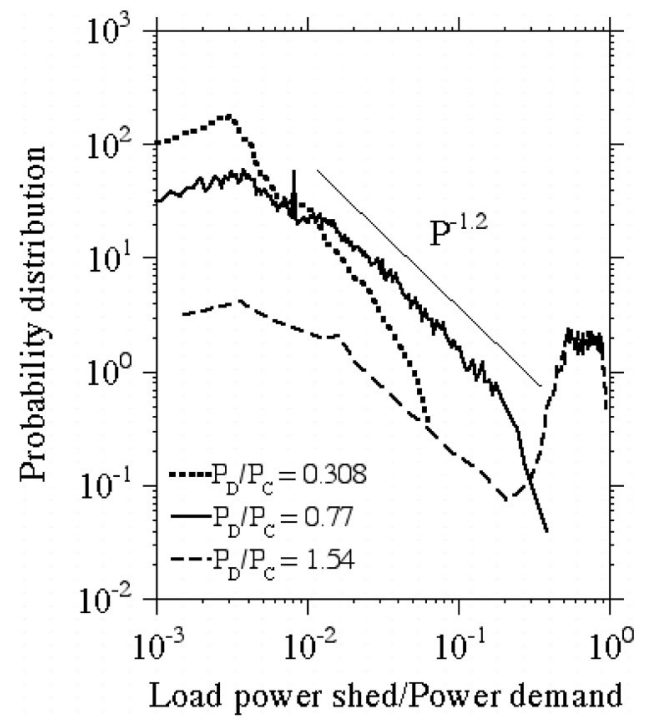

FIG. 12. PDF of the normalized load shed for a tree 382-node tree network for different levels of the power demand.

We have done a sequence of calculations with $\gamma=1.9$ and $P_{D} / P_{C}$ varying between 0.3 and 1.5 for the tree network with 382 nodes. For each set of parameters, we have considered 60000 cases by random variation of the loads. This number of cases has allowed accumulating enough statistics to calculate the probability distribution function (PDF) of the amount of load shed. Load power shed is one measure of blackout size.

In Fig. 12, we have plotted the PDF of the load power shed normalized to the power demand for three values of $P_{D} / P_{C}$. Well below the critical point, the PDF is peaked at low values of the power shed and has a tail falling off as the -2 power of the load shed. This type of PDF is a consequence of the network structure we have considered. It can be shown analytically that a single line failure leads to a blackout PDF decaying as $P^{-2}$. High above the critical point, it has a highly peaked form with mean value at high values of the power shed. As the power demand reaches to the critical point, the PDF develops a power tail with a decay index close to -1 . This is indicative that some of these transitions have the properties of critical transitions. ${ }^{20,21}$ For the parameters used in Fig. 12, the level of fluctuations is high enough to reach the critical value for a power demand of $30 \%$ below the generator limit. We have included a probability of a random line failure $p_{0}=10^{-4}$. Because of the finite size of the system, there is an exponential cutoff in the PDF. To positively identify the power tail region, it is important to consider large networks and do finite size scaling of the results. In Fig. 13, we compare the PDF close to the critical point for a 46-node tree network with the 382-node tree network. We can see that the algebraic region of the tail expands as the network size increases.

Similar behavior has also been observed in a simple analytic model of cascading failure and in a power transmission system model that represents cascading failure because of hidden failures of the protection system. ${ }^{22}$ 


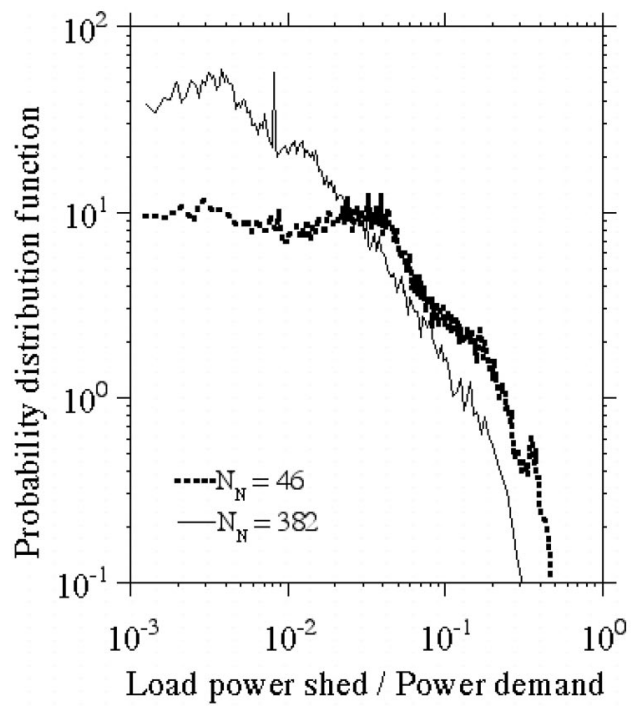

FIG. 13. Comparison of the PDFs of the normalized load shed 46-node and 382-node tree networks.

\section{ANALYSIS OF A MORE REALISTIC NETWORK}

The properties described in the previous sections for the ideal tree networks also apply to more realistic networks. However, it is more difficult to make a proper identification of the transition points in more realistic networks because of the variations in line limits and line flows. Moreover, for more realistic networks, the total power demand is no longer a unique parameter to vary in order to produce the critical points, and a proper parameter representation requires a multidimensional space.

We have used one of the standard networks for power system studies, the IEEE 118 bus network, ${ }^{14}$ as an example of a more realistic network. The values of the parameters used in these calculations are given in Ref. 14. Figure 14

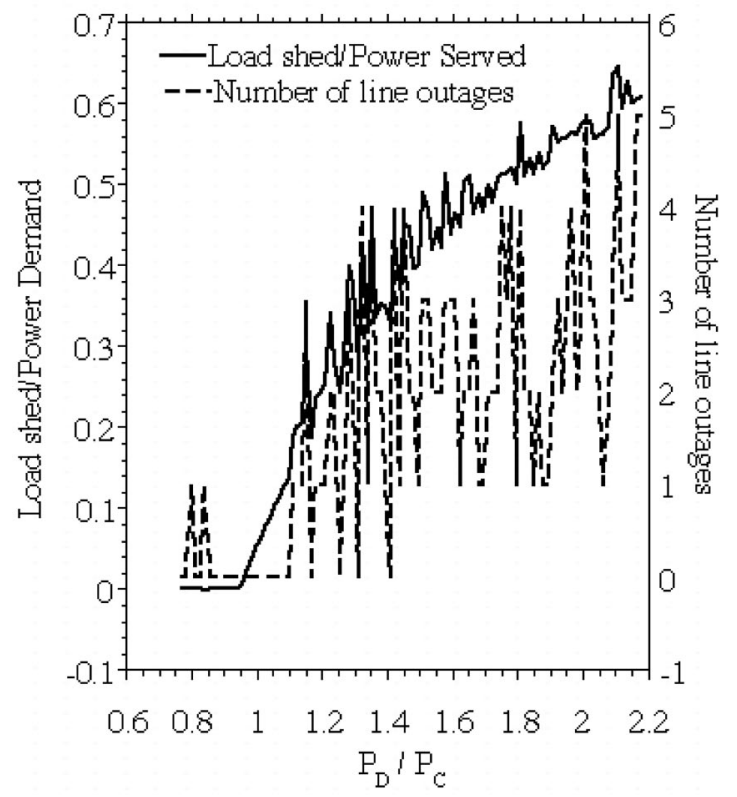

FIG. 14. Normalized power shed and number of outaged lines for the IEEE 118 bus network as a function of power demand.

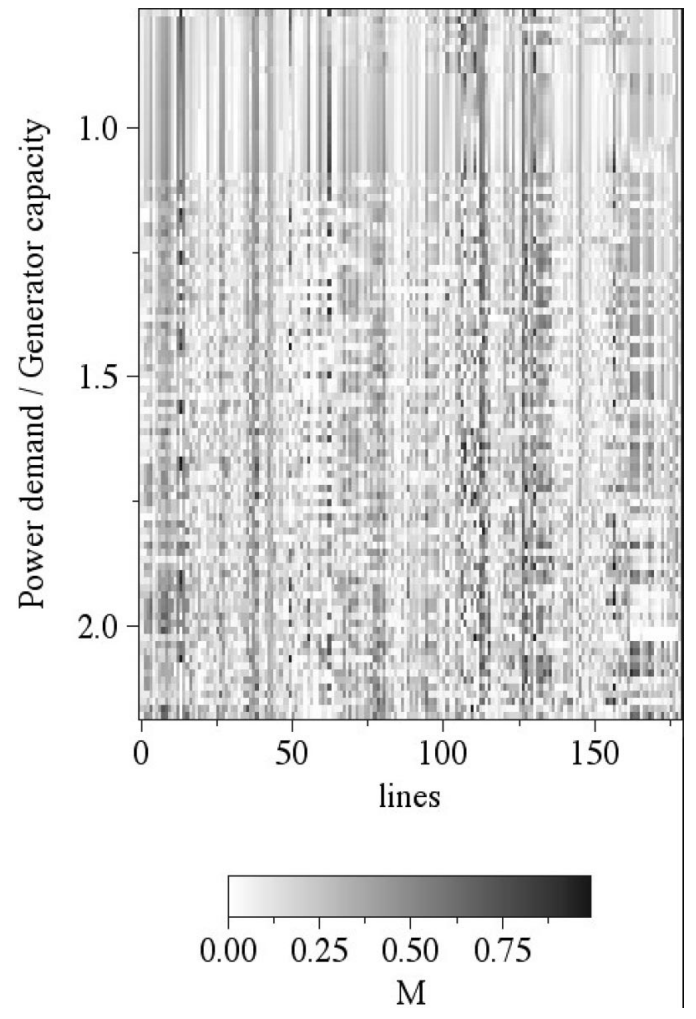

FIG. 15. Two-dimensional plot of the fraction of overload of the lines in the IEEE 118 bus network as a function of line and power demand.

shows a power demand scan for the IEEE 118-bus network similar to the scan for the ideal network in Fig. 5. In particular, Fig. 14 shows the load shed and the number of line outages as a function of the power demand. There is a critical point caused by the maximum generator power slightly below $P_{D} / P_{C}=1.0$. However, the $M_{\max }=1$ critical points are spread over a large range of power demand, even below $P_{D} / P_{C}=1.0$.

In Fig. 15, we have plotted $M_{i j}$ in a two-dimensional plot as a function of power demand and line number. Figure 15 is the analog to Fig. 9, and we can see that Fig. 15 shows more structure than Fig. 9. That should be expected because the fraction of overloads for each line have different values, and they reach the limit for very different levels of power demand. However, there is a continuous dependence of the solutions with power demand for $P_{D} / P_{C}<1.1$, except for a narrow band with one line outage. When $P_{D} / P_{C}=1.1$, there are many transitions associated with $M_{\max }=1$. In this region, the solutions lie in narrow bands that change in an erratic way because different lines reaching their power flow limits. Thus, we observe the same qualitative behavior of the solutions as for the ideal networks. However, the limits associated with the line limits are now spread over a range of values of the power demand, and the associated jumps in the loads shed are individually smaller than in Fig. 11, in which many of these limits happen at the same power demand level.

When the load scan is done allowing fluctuation of the loads, we can calculate the probability distribution function of blackouts for different values of the mean load demand. In this case, the PDF at low values of the power demand is 


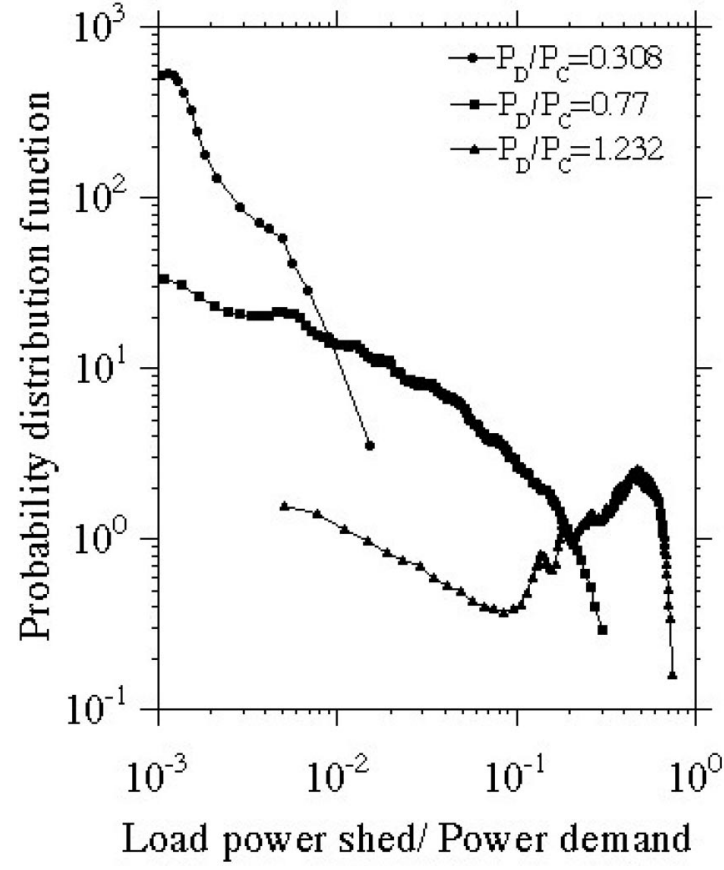

FIG. 16. PDF of the normalized load shed for the IEEE 118 bus network for different levels of power demand.

close to a Gaussian function, because a single line failure does not induce large blackouts. As was the case in the ideal networks, we observe in Fig. 16 the appearance of a power tail in the proximity of the critical points. Because the network has only 118 nodes, the decay index is difficult to determine, as we discussed in relation to the comparison shown in Fig. 13.

\section{CONCLUSIONS}

The power transmission model discussed in this paper has two types of transitions in its cascading failure blackouts as the load power demand is increased. The first type of transition is because of the limit on total generator capacity. The second type of transition is due to the power flow limits of the network lines. If we characterize the blackouts by the amount of load power shed, we can see that at the first transition point the power shed starts increasing with the power demand. Its value is continuous at this point, but its derivative is discontinuous. Therefore, it has the characteristic properties of a second order transition.

The second type of transition is characterized by a sudden jump in the power shed. Both the value and the derivative of the power shed as a function of the power demand are discontinuous at these transition points. These transitions, caused by limitations in the transmission lines, are similar to a first-order transition. They can lead to solutions of the system that appear to be erratic as the power demand changes. There are many narrow bands of solutions and the edge of each band corresponds to a line limit.

Some of these transition points have the characteristic properties of a critical transition. That is, when the load power demand is close to a critical value, the probability distribution function of the blackout size has an algebraic tail, and, at the critical loading, the risk for blackouts increases sharply.

The general results found for ideal, homogeneous tree networks have also been reproduced in the more realistic inhomogeneous case of the IEEE 118-bus network. These calculations confirm the robustness of the results.

Analyses of 15 years of North American blackout data show a probability distribution of blackout size that has a power tail ${ }^{3,4,23}$ similar to the power tails found in this paper near the critical transitions. This analysis suggests that the North American power system may be operated close to these critical transitions. Such operation may be the results of competing forces, such as the secular increase of the power demand and the upgrading of the power system as a response to this demand leading to a complex dynamical behavior. These competing forces may lead to a complex dynamics evolution of the power system. This possibility is under investigation. ${ }^{9-11}$

\section{ACKNOWLEDGMENTS}

Part of this research has been carried out at Oak Ridge National Laboratory, managed by UT-Battelle, LLC, for the U.S. Department of Energy under Contract No. DE-AC0500OR22725. I.D. and D.N. gratefully acknowledge support in part from NSF Grants Nos. ECS-0085711 and ECS0085647. I.D. and B.A.C. gratefully acknowledge coordination of part of this work by the Consortium for Electric Reliability Technology Solutions and funding in part by the Assistant Secretary for Energy Efficiency and Renewable Energy, Office of Power Technologies, Transmission Reliability Program of the U.S. Department of Energy under Contract No. 9908935 and Interagency Agreement DEA1099EE35075 with the National Science Foundation.

\section{APPENDIX: THE dc POWER FLOW MODEL}

In the dc power flow model, the bus (node) voltages are $60 \mathrm{~Hz}$ phasors specified by complex number magnitude and phase. Uniform voltage magnitudes normalized to 1 are assumed so that the voltage magnitude is 1 and the voltage phase is $\theta_{i}$, where $\theta_{i}$ is the voltage angle at bus $i$. The reference bus has voltage angle zero. Then an $n$-dimensional vector $\Theta$ of voltage angles can be defined including the zero angle of the reference bus.

The transmission lines are characterized by transmission line susceptance, $b_{i j}$. Since transmission line resistance is neglected, $b_{i j}=1 / z_{i j}$ where $z_{i j}$ is the line inductance. The $N_{l} \times N_{l}$ matrix $B$ is defined by

$$
\begin{aligned}
B_{i i} & =\sum_{\text {node } j} \sum_{\text {connected to node } i} b_{i j}, \\
B_{i j} & =-b_{i j} .
\end{aligned}
$$

The transmission line susceptance accounts for susceptance of the transmission line as well as the susceptance of transformers in the line. 
From standard ac circuit equations ${ }^{10}$ and after linearization we have the following relation between the power input at the nodes and the voltage angle

$$
P=B \Theta \text {. }
$$

Here, $P$ is the vector defined in Sec. II. The matrix $B$ must be singular ( $B$ has rank $N_{N}-1$ ) because of the constraint $\sum_{k=0}^{N_{G}} P_{k}=0$. Inverting Eq. (A2), allowing for the constraint and using the zero angle of the reference bus, we obtain the voltage phase in terms of the power generator of each node, $\Theta=X P$.

The flow on the line connecting bus $i$ to bus $j$ is $F_{i j}$ $=b_{i j}\left(\theta_{i}-\theta_{j}\right)$. Therefore, combining all these relation we obtain Eq. (1).

${ }^{1}$ D. N. Ewart, IEEE Spectrum 36, 1 (1978).

${ }^{2}$ Information on electric systems disturbances in North America can be downloaded from the NERC website at http://www.nerc.com/dawg/ database.html

${ }^{3}$ B. A. Carreras, D. E. Newman, I. Dobson, and A. B. Poole, "Initial evidence for self-organized criticality in electric power system blackouts," 33rd Hawaii International Conference on System Sciences, Maui, Hawaii, January 2000.

${ }^{4}$ B. A. Carreras, D. E. Newman, I. Dobson, and A. B. Poole, "Evidence for self-organized criticality in electric power system blackouts," 34th Hawaii International Conference on System Sciences, Maui, Hawaii, January 2001.

${ }^{5}$ J. Chen, J. S. Thorp, and M. Parashar, "Analysis of electric power disturbance data," 34th Hawaii International Conference on System Sciences, Maui, Hawaii, January 2001.

${ }^{6}$ R. Billington and R. N. Allan, Reliability Assessment of Large Electric Power Systems (Kluwer Academic, Boston, 1987).

${ }^{7}$ S. H. Strogatz, Nature (London) 410, 268 (2001).

${ }^{8}$ R. Albert and A.-L. Barabási, Rev. Mod. Phys. 74, 47 (2002).
${ }^{9}$ I. Dobson, B. A. Carreras, V. Lynch, and D. E. Newman, "An initial model for complex dynamics in electric power system blackouts," 34th Hawaii International Conference on System Sciences, Maui, Hawaii, January 2001.

${ }^{10}$ B. A. Carreras, V. E. Lynch, M. L. Sachtjen, I. Dobson, and D. E. Newman, "Modeling blackout dynamics in power transmission networks with simple structure," 34th Hawaii International Conference on System Sciences, Maui, Hawaii, January 2001.

${ }^{11}$ B. A. Carreras, V. E. Lynch, I. Dobson, and D. E. Newman, "Dynamics, criticality and self-organization in a model for blackouts in power transmission systems," 35th Hawaii International Conference on System Sciences, Hawaii, January 2002.

${ }^{12}$ A. J. Wood and B. F. Wollenberg, Power Generation, Operation and Control (Wiley, New York, 1984).

${ }^{13}$ K. Nagel and M. Paczuski, Phys. Rev. E 51, 2909 (1995).

${ }^{14}$ The IEEE 118 bus network model is a standard test system; see http://www.ee.washington.edu/research/pstca/

${ }^{15}$ M. Biehl and A. Mietzner, Europhys. Lett. 24, 421 (1993).

${ }^{16}$ B. Stott and E. Hobson, IEEE Trans. Power Appar. Syst. PAS-97, 1713 (1978).

${ }^{17}$ B. Stott and E. Hobson, IEEE Trans. Power Appar. Syst. PAS-97, 1721 (1978).

${ }^{18}$ B. Stott and J. L. Marinho, IEEE Trans. Power Appar. Syst. PAS-98, 837 (1979).

${ }^{19}$ W. H. Press, B. P. Flannery, S. A. Teukolsky, and W. T. Vetterling, $\mathrm{Nu}$ merical Recipes in $C$ (Cambridge University Press, Cambridge, 1988).

${ }^{20}$ J. J. Binney, N. J. Dowrick, A. J. Fisher, and M. E. J. Newman, The Theory of Critical Phenomena (Clarendon, Oxford, 1992).

${ }^{21}$ N. Goldenfeld, Lectures on Phase Transitions and the Renormalization Group (Addison-Wesley, New York, 1992).

${ }^{22}$ I. Dobson, J. Chen, J. S. Thorp, B. A. Carreras, and D. E. Newman, "Examining criticality of blackouts in power system models with cascading events," 35th Hawaii International Conference on System Sciences, Hawaii, Hawaii, January 2002.

${ }^{23}$ B. A. Carreras, D. E. Newman, I. Dobson, and A. B. Poole, "Evidence for self-organized criticality in a time series of electric power system blackouts," submitted to IEEE Trans. Power Appar. Syst. 